\title{
Association of MTHFR C677T and AI298C polymorphisms with oral cancer susceptibility: evidence from a meta-analysis
}

This article was published in the following Dove Press journal:

OncoTargets and Therapy

10 January 2017

Number of times this article has been viewed

\author{
Sui Jiang ${ }^{1, *}$ \\ Jin-Dong $X \mathbf{u}^{2}$ \\ Zhen-Jian Zhuo ${ }^{3}$ \\ Zhu-Ming $\mathrm{Hua}^{2}$,* \\ 'Department of Oral and \\ Maxillofacial Surgery, ${ }^{2}$ Department of \\ Anesthesiology, Guangdong General \\ Hospital, Guangdong Academy \\ of Medical Sciences, Guangzhou, \\ Guangdong, ${ }^{3}$ School of Chinese \\ Medicine, Faculty of Medicine, The \\ Chinese University of Hong Kong, \\ Hong Kong, People's Republic \\ of China \\ *These authors contributed equally \\ to this work
}

Correspondence: Sui jiang

Department of Oral and Maxillofacial

Surgery, Guangdong General Hospital,

Guangdong Academy of Medical Sciences,

106 Zhongshan Er Rd, Guangzhou

510080, Guangdong, People's Republic

of China

Tel +86 2083827812

Fax +86 2083827818

Email suijiang08@sina.com

Zhu-Ming Hua

Department of Anesthesiology,

Guangdong General Hospital, Guangdong

Academy of Medical Sciences, 106

Zhongshan Er Rd, Guangzhou 510080 ,

Guangdong, People's Republic of China

Tel +862083827812

Fax +86 2083827818

Email mdhuazhuming@gmail.com
Abstract: Methylenetetrahydrofolate reductase (MTHFR) is a central enzyme involved in folate metabolism and plays an important role in DNA synthesis and methylation. Several studies have been conducted to illustrate the associations between MTHFR C677T and A1298C polymorphisms with oral cancer susceptibility; however, the results are inconsistent. Therefore, we conducted an updated meta-analysis to obtain a more reliable estimation of the associations. We retrieved eligible studies from PubMed, EMBASE, and CBM databases through September 2016. Ultimately, pooled analyses involved 10 studies with 1443 cases and 1640 controls for the C677T polymorphism, as well as five studies with 973 cases and 1024 controls for the A1298C polymorphism. Risk estimates were presented as odds ratios (ORs) and 95\% confidence intervals (95\% CIs). Pooled results indicated that neither C677T nor A1298C polymorphism was associated with oral cancer susceptibility. However, a borderline significant association was detected between MTHFR C677T polymorphism and a decreased oral cancer risk (homozygous model: $\mathrm{OR}=0.71,95 \% \mathrm{CI}=0.50-1.00)$ in hospital-based studies. Our results suggested that MTHFR C677T and A1298C polymorphisms might not be associated with oral cancer risk. However, more evidence is needed to further confirm these findings in the future.

Keywords: $M T H F R$, polymorphisms, oral cancer, susceptibility, meta-analysis

\section{Introduction}

Cancer is a major public health burden. An estimated 14.1 million new cancer cases and 8.2 million cases of cancer deaths arose worldwide, according to the data from GLOBOCAN 2012. ${ }^{1}$ Oral cancer, a subtype of head and neck cancer, is one of the most frequently diagnosed human malignancies in the world. Lines of evidence suggest that smoking, ${ }^{2}$ alcohol drinking, ${ }^{3}$ insufficient fruits and vegetables intake, ${ }^{4}$ and oral HPV infection $^{5}$ are well-established risk factors for oral cancer. However, only a small part of exposed people eventually develop oral cancer. One acceptable explanation for this phenomenon is that genetic variations also modify individuals' risk of developing cancer, beside health-risk environmental exposures and lifestyles. ${ }^{6}$

Previous studies demonstrated that folic acid deficiency resulting from low intake of fruits and vegetables increases the risk of developing cancer. ${ }^{7,8}$ In humans, folate provides the majority of methyl groups for de novo deoxynucleoside synthesis and intracellular methylation reactions. ${ }^{9}$ Folate deficiency may lead to uracil misincorporation during DNA synthesis, thus impairing the stability of DNA. ${ }^{10}$ Folate-metabolizing enzyme, methylenetetrahydrofolate reductase (MTHFR), is a key component in folic acid cycle and DNA synthesis. MTHFR catalyzes the irreversible reduction of 5,10methylenetetrahydrofolate to 5-methyltetrahydrofolate, donating a methyl group for the 
methylation of homocysteine to methionine. ${ }^{11}$ Dysfunction of MTHFR may disrupt the dynamic equilibrium of methyl pool and consequently influence the deoxynucleoside synthesis and intracellular methylation reactions.

The human MTHFR gene is located on chromosome 1p36.3. By now, two common nonsynonymous MTHFR single-nucleotide polymorphisms (SNPs), C677T (Ala222Val, rs1801133) and A1298C (Glu429Ala, rs1801131), have been widely investigated. ${ }^{12,13}$ It has been shown that C677T and A1298C polymorphisms are associated with the risk of several types of cancer, including breast cancer, ${ }^{14}$ colorectal cancer, ${ }^{15}$ non-Hodgkin's lymphoma, ${ }^{16}$ and cervical cancer. ${ }^{17}$ Different research groups have extensively assessed the association of C677T and A1298C polymorphisms with oral cancer risk, yet produce discrepant results. ${ }^{18,19}$ Four metaanalyses regarding such association have been conducted previously but somehow fail to establish reliable relationship between the two MTHFR SNPs and oral cancer risk. ${ }^{20-23}$ Therefore, we performed this evidence-based quantitative meta-analysis to provide a precise evaluation of the associations by including more eligible investigations.

\section{Materials and methods}

\section{Literature search}

We performed a comprehensive literature search in the PubMed and EMBASE, as well as Chinese databases (China National Knowledge infrastructure and WANFANG). No language limitation was applied in the literature search. The last research was updated in September 2016. The following search terms were adopted: "MTHFR or methylenetetrahydrofolate reductase", "polymorphism or polymorphisms or SNP or single nucleotide polymorphism or variant", and "oral cancer or oral carcinoma". We also manually searched references listed in the retrieved articles after carefully reviewing. The latest or the largest study was included in the final meta-analysis, if there exist two or more articles containing overlapping data.

\section{Inclusion/exclusion criteria}

We used the following standards to select eligible articles: 1) studies evaluating the association of MTHFR C677T and A1298C polymorphisms with oral cancer risk; 2) casecontrol design; 3) sufficient data provided to compute odds ratios (ORs) and 95\% confidence intervals (95\% CIs); and 4) in the controls, genotype frequency of $M T H F R$ C677T, A1298C, or other SNPs in agreement with HardyWeinberg equilibrium (HWE). Exclusion criteria were as follows: 1) case-only studies or case reports; 2) reviews or meta-analyses; 3) studies without detailed genotyping data; and 4) duplicate publications.

\section{Data extraction}

Two investigators (Zhu-Ming Hua and Zhen-Jian Zhuo) independently extracted data from the eligible studies. For conflicting results, a consensus was achieved after thorough discussion. The following characteristics were selectively extracted from each study: first author's surname, year of publication, country, ethnicity, the source of controls, genotyping methods, and genetic distribution of cases and controls. The stratification analysis was conducted by ethnicity (Asians and Caucasians) and control source (hospital based and population based).

\section{Statistical methods}

Chi-square test was used to check the genotype frequency of SNPs among the controls, with $P<0.05$ suggesting a departure from HWE. The strength of association between MTHFR C677T and A1298C polymorphisms and oral cancer risk was assessed by calculating pooled ORs and 95\% CIs. The following five genetic models were applied: homozygote model (C677T: TT vs CC; A1298C: CC vs AA), heterozygote model (C677T: CT vs CC; A1298C: AC vs AA), recessive model (C677T: TT vs CT/CC; A1298C: CC vs AC/AA), dominant model (C677T: CT/TT vs CC; A1298C: AC/CC vs AA), and allele contrast (C677T: T vs C; A1298C: C vs A). Stratification analyses were also performed by ethnicity (Asians and Caucasians) and source of control (hospital based and population based). We performed chi-square-based $\mathrm{Q}$ statistic test and inconsistency index statistics $\left(I^{2}\right)$ to calculate between-study heterogeneity. If the studies were found to be homogeneous (with $P^{\text {het }}>0.10$ or $I^{2}<50 \%$ ), ORs were estimated using the fixed-effects model (the Mantel-Haenszel method). Otherwise, the random-effects model (the DerSimonian and Laird method) was adopted. Sensitivity analysis was performed by sequentially removing one study at a time and reanalyzing the pooled estimates, in order to exclude single studies that have significant influences on the overall ORs. Furthermore, potential publication bias was estimated by Begg's funnel plot as well as Egger's linear regression test. An asymmetric plot and a $P$-value $<0.05$ indicated the existence of publication bias. All the data management and statistical analysis were performed using STATA 11.0 software (Stata Corporation, College Station, TX, USA). All statistical tests were two-sided. A $P$-value of $<0.05$ was set as a significant threshold. 


\section{Trial sequential analysis (TSA)}

To avoid the random errors caused by dispersed data and repeated significance testing, we performed the TSA to obtain a more reliable conclusion..$^{24} \mathrm{We}$ only applied TSA to MTHFR C677T polymorphism, but not A1298C polymorphism, as the latter involves less than six studies. We calculated the required information size and estimated the sample size needed to make a robust conclusion. The required information size was evaluated by considering an overall type I error (a) of 5\% and type II error (b) of $20 \%$. Based on required information size as well as risk for type I and type II errors, TSA monitoring boundaries were constructed. If the cumulative Z-curve (blue line) crosses a TSA monitoring boundary (red lines sloping inward) before the required information size is reached, the robustness of evidence may be confirmed and no further trials are necessary. Otherwise, the evidence is inadequate to get a robust conclusion and it is necessary to continue to do trials. TSA software version 0.9 beta was used.

\section{Results}

\section{Selection of studies}

The identification and selection of studies are graphically presented in Figure 1. A total of 41 potentially relevant published records were initially retrieved from PubMed and EMBASE, and only one article was identified from Chinese databases. Among them, 25 articles were excluded after screening the titles and abstracts, and 17 remaining studies were further subjected to full-text assessment. Among them, seven studies were removed for the following reasons: five were meta-analyses or reviews, ${ }^{6,20-23}$ one was duplication publication, ${ }^{25}$ and one lacked description of oral cancer. ${ }^{26}$ Finally, 10 publications were included in the meta-analysis, of which 10 studies involved C677T polymorphism ${ }^{18,19,27-34}$ and five involved A1298C polymorphism. . $8,19,28,29,32$

\section{Study characteristics}

The study characteristics of the final selected studies are shown in Table 1. Ten studies with 1443 cases and 1640 controls for the C677T polymorphism were ultimately included in the meta-analysis. Among them, seven studies were conducted in Caucasians and three in Asians. As to the source of control, four were population-based studies and six were hospital based. Moreover, there were five studies for the A1298C polymorphism with 973 cases and 1024 controls. Of these studies, three were conducted in Caucasians and two in Asians. All of them were hospital-based design.

\section{Meta-analysis results}

The main results of the current meta-analysis are listed in Table 2 and Figure 2. As for C677T polymorphism, no

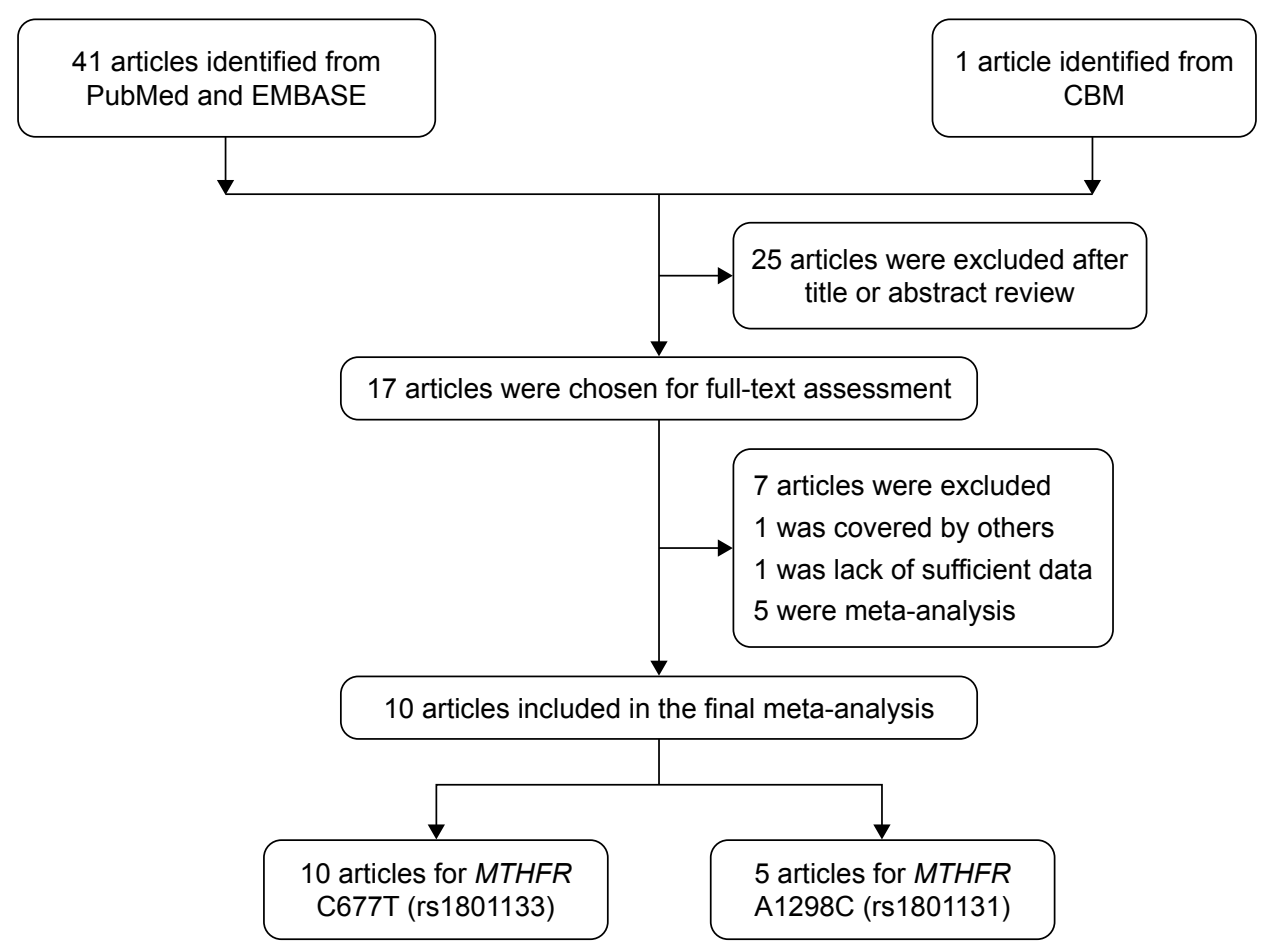

Figure I Flow diagram of the included studies for the associations between MTHFR C677T and AI298C polymorphisms and oral cancer susceptibility. Abbreviation: MTHFR, methylenetetrahydrofolate reductase. 
Table I Characteristics of studies included in this meta-analysis

\begin{tabular}{|c|c|c|c|c|c|c|c|c|c|c|c|c|c|c|c|}
\hline Surname & Year & Country & Ethnicity & Control & Genotype & Case & & & & Cont & trol & & & MAF & HWE \\
\hline C677T polymorphism & & & & & & CC & CT & TT & All & CC & CT & TT & All & & \\
\hline Weinstein ${ }^{33}$ & 2002 & Puerto Rico & Caucasian & PB & PCR-RFLP & 67 & 53 & 15 & 135 & 69 & 62 & 15 & 146 & 0.32 & 0.846 \\
\hline Capaccio $^{28}$ & 2005 & Italy & Caucasian & $\mathrm{HB}$ & PCR & 18 & 33 & 14 & 65 & 36 & 46 & 18 & 100 & 0.41 & 0.623 \\
\hline Solomon ${ }^{30}$ & 2008 & India & Asian & PB & PCR-RFLP & 48 & 55 & 23 & 126 & 48 & 42 & 10 & 100 & 0.31 & 0.855 \\
\hline Sailasree 29 & 2011 & India & Asian & $\mathrm{HB}$ & PCR-RFLP & 92 & 8 & 1 & 101 & 108 & 29 & 1 & 138 & 0.11 & 0.527 \\
\hline Supic ${ }^{31}$ & 2011 & Serbia & Caucasian & PB & PCR-RFLP & 50 & 32 & 14 & 96 & 80 & 66 & 16 & 162 & 0.30 & 0.661 \\
\hline Tsai ${ }^{32}$ & 2011 & China & Asian & $\mathrm{HB}$ & PCR & 391 & 186 & 43 & 620 & 322 & 236 & 62 & 620 & 0.29 & 0.058 \\
\hline Vylliotis ${ }^{34}$ & 2013 & Greek/Germany & Caucasian & PB & PCR-RFLP & 28 & 76 & 6 & 110 & 45 & 65 & 10 & 120 & 0.35 & 0.044 \\
\hline Bezerra $^{27}$ & 2014 & Brazil & Caucasian & $\mathrm{HB}$ & PCR-RFLP & 19 & 12 & I & 32 & 48 & 41 & I & 90 & 0.24 & 0.016 \\
\hline Barbosa $^{19}$ & 2015 & Brazil & Caucasian & $\mathrm{HB}$ & PCR-RFLP & 50 & 45 & 6 & 101 & 50 & 41 & 11 & 102 & 0.31 & 0.555 \\
\hline Miri-Moghaddam ${ }^{18}$ & 2016 & Iran & Caucasian & $\mathrm{HB}$ & PCR & 34 & 21 & 2 & 57 & 47 & 14 & 1 & 62 & 0.13 & 0.971 \\
\hline AI298C polymorphism & & & & & & AA & AC & CC & All & $\mathbf{A A}$ & AC & CC & All & & \\
\hline Capaccio 28 & 2005 & Italy & Caucasian & $\mathrm{HB}$ & PCR & 31 & 28 & 6 & 65 & 44 & 51 & 6 & 101 & 0.31 & 0.076 \\
\hline Sailasree ${ }^{29}$ & 2011 & India & Asian & $\mathrm{HB}$ & PCR-RFLP & 37 & 74 & 19 & 130 & 46 & 59 & 34 & 139 & 0.46 & 0.088 \\
\hline Tsai ${ }^{32}$ & 2011 & China & Asian & $\mathrm{HB}$ & PCR & 407 & 192 & 21 & 620 & 393 & 198 & 29 & 620 & 0.21 & 0.528 \\
\hline Barbosa ${ }^{19}$ & 2015 & Brazil & Caucasian & $\mathrm{HB}$ & PCR-RFLP & 60 & 36 & 5 & 101 & 53 & 44 & 5 & 102 & 0.26 & 0.275 \\
\hline Miri-Moghaddam ${ }^{18}$ & 2016 & Iran & Caucasian & $\mathrm{HB}$ & PCR & 30 & 26 & I & 57 & 34 & 26 & 2 & 62 & 0.24 & 0.259 \\
\hline
\end{tabular}

Abbreviations: MAF, minor allele frequency; HWE, Hardy-Weinberg equilibrium; PB, population based; HB, hospital based; PCR, polymerase chain reaction; PCR-RFLP, polymerase chain reaction-restriction fragment length polymorphism.

significant association with overall oral cancer risk was observed under all the five genetic models: homozygote model (TT vs CC: $\mathrm{OR}=0.92,95 \% \mathrm{CI}=0.71-1.20$ ), heterozygous model (CT vs CC: $\mathrm{OR}=0.98,95 \% \mathrm{CI}=0.72-1.33$ ), recessive model (TT vs $\mathrm{CT} / \mathrm{CC}$ : $\mathrm{OR}=0.95,95 \% \mathrm{CI}=0.74-1.23$ ), dominant model (CT/TT vs CC: $\mathrm{OR}=1.00,95 \% \mathrm{CI}=0.74$ 1.37), and allele model ( $\mathrm{T}$ vs $\mathrm{C}$ : $\mathrm{OR}=1.00,95 \% \mathrm{CI}=0.80$ 1.27). Stratified analysis by ethnicity did not revealed any significant association between C677T polymorphism and oral cancer risk among either ethnic group. In terms of source of controls, only a borderline significant association was detected between C677T polymorphism and a decreased oral cancer risk under the homozygous model $(\mathrm{OR}=0.71$, 95\% CI $=0.50-1.00$ ) in hospital-based studies.

Regarding the A1298C polymorphism, no association with overall oral cancer susceptibility was found (CC vs AA: $\mathrm{OR}=0.76,95 \% \mathrm{CI}=0.52-1.13 ; \mathrm{AC}$ vs $\mathrm{AA}: \mathrm{OR}=0.97,95 \%$ $\mathrm{CI}=0.80-1.17$; $\mathrm{CC}$ vs AC/AA: $\mathrm{OR}=0.71,95 \% \mathrm{CI}=0.49-1.03$; $\mathrm{AC} / \mathrm{CC}$ vs $\mathrm{AA}: \mathrm{OR}=0.93,95 \% \mathrm{CI}=0.77-1.11$; and $\mathrm{C}$ vs $\mathrm{A}$ : $\mathrm{OR}=0.90,95 \% \mathrm{CI}=0.78-1.05$ ). Similarly, we could not observe any association between A1298C polymorphism and oral cancer risk in the stratified analysis by ethnicity.

\section{Heterogeneity and sensitivity analysis}

As shown in Table 2, substantial heterogeneities existed among the investigations for the MTHFR C677T polymorphism and oral cancer risk under the heterozygous $(P=0.001)$ and dominant $(P<0.001)$ models, as well as allele contrast $(P=0.001)$. Thus, the random-effects model was applied to calculate the pooled ORs and 95\% CIs for these genetic models. The fixed-effect model was adopted for other two genetic models without heterogeneity.

The sequential leave-one-out sensitivity analysis was applied to investigate the influence of a single study on the pooled ORs. The similar recalculated ORs were obtained after the omission of each individual study, indicating the reliability and credibility of our results (data not shown).

\section{Publication bias}

We performed Begg's funnel plot and quantitative Egger's linear regression test to evaluate the publication bias. The shape of the Begg's funnel plots appeared to be symmetrical for C677T and A1298C polymorphisms. Moreover, we did not observe significant publication bias as suggested by Egger's test.

\section{TSA}

In this meta-analysis, 10 studies with 3083 individuals were included to investigate the association of $\mathrm{C} 677 \mathrm{~T}$ polymorphism with oral cancer susceptibility. The TSA for C677T polymorphism showed that the cumulative Z-curve did not cross the trial monitoring boundary before reaching the required information size, suggesting that additional trials were needed to further verify the conclusion (Figure 3). TSA was not applicable for A1298C polymorphism, since the number of eligible studies was too small.

\section{Discussion}

In this meta-analysis, we analyzed the association of MTHFR C677T and A1298C polymorphisms with oral cancer risk. 


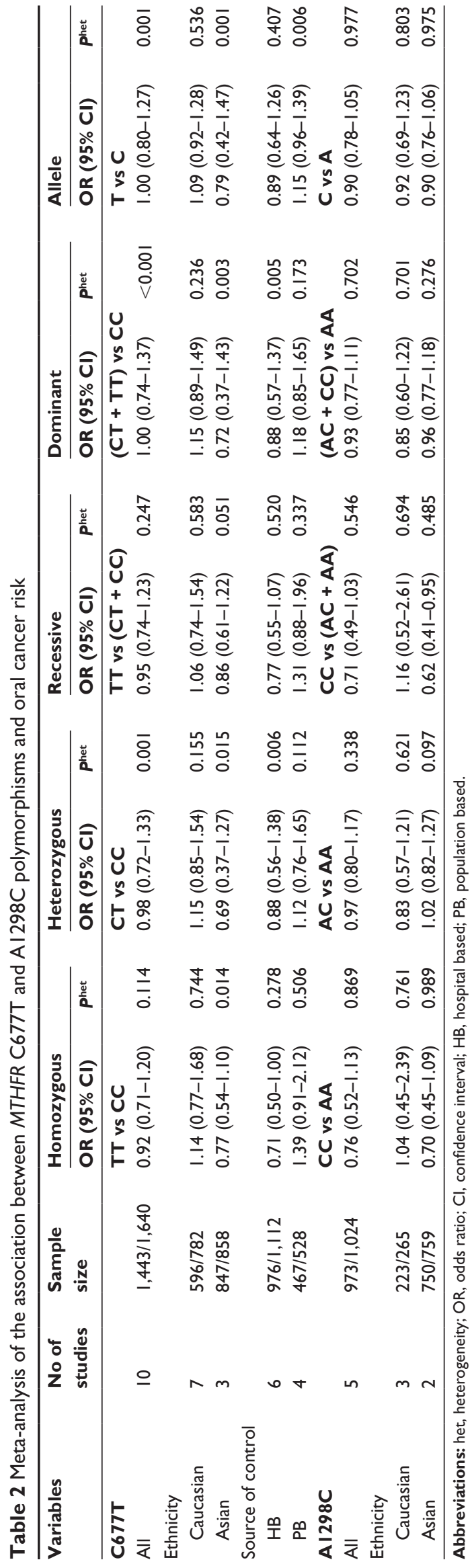

No significant association with oral cancer was observed for the two SNPs under all the five genetic models. In addition, stratified analyses by ethnicity revealed null association in Caucasians and Asians. However, we detect a borderline significant association of C677T polymorphism with a decreased oral cancer risk under the homozygous model in studies with hospital-based design. To the best of our knowledge, this is the largest meta-analysis on this topic, including all the eligible epidemiological studies in English and Chinese.

The MTHFR C677T polymorphism, a C-to-T transition at position 677, induces the substitution of valine for alanine in codon $222 .{ }^{35}$ It is a functional genetic variation. Studies elucidated that the variant $\mathrm{T}$ alleles of this MTHFR polymorphism reduced the enzyme activity to nearly $50 \%$, compared to the wild-type $\mathrm{C}$ allele. ${ }^{36}$ It was reported that $\mathrm{C} 677 \mathrm{~T}$ polymorphism was associated with a decreased risk in prostate cancer, ${ }^{37}$ colorectal carcinoma, ${ }^{38}$ leukemia, ${ }^{39}$ and malignant lymphoma. ${ }^{40}$ However, studies on breast cancer, ${ }^{41}$ gastric cancer, ${ }^{42}$ hepatocellular cancer, ${ }^{43}$ and cervical cancer ${ }^{44}$ have found an increased cancer risk associated with the variant $\mathrm{T}$ allele. Although numerous studies have been performed, the associations of MTHFR C677T and A1298C polymorphisms with oral cancer susceptibility remain contradictory, in part, due to the limited sample size. By now, four metaanalyses were published regarding association between these two MTHFR polymorphisms and oral cancer risk, with three articles written in English and one in Chinese.

Zhuo et $\mathrm{al}^{20}$ conducted the first meta-analysis including six studies on C677T polymorphism and two studies on A1298C polymorphism in 2012. They found no impact of C677T polymorphism on oral cancer susceptibility in the pooled analysis. However, the same study observed the significant association of A1298C polymorphism with decreased oral cancer susceptibility under the recessive model. In 2013, Xu et al ${ }^{22}$ performed an updated meta-analysis in Chinese to identify the role of C677T polymorphism in oral cancer risks with seven studies. They failed to find the association between C677T polymorphism and oral cancer risk. Subgroup analysis based on the source of control revealed decreased susceptibility to cancer among hospital-based population. After that, Jia et $\mathrm{al}^{21}$ performed another meta-analysis including 1318 cases and 1817 controls from seven studies. They found a borderline association between C677T polymorphism and oral cancer risk under the heterogeneous model. Moreover, stratified analysis demonstrated ethnic- and control design-specific associations with $\mathrm{C} 677 \mathrm{~T}$ polymorphism, ie, $\mathrm{T}$ allele was identified as a protective allele against oral cancer in Asians and in hospital-based studies. In a recent study conducted 


\begin{tabular}{|c|c|c|}
\hline Study ID & OR $(95 \% \mathrm{CI})$ & $\%$ weight \\
\hline Weinstein et $\mathrm{al}^{33}$ & $1.09(0.51,2.33)$ & 10.46 \\
\hline Capaccio et al ${ }^{28}$ & $1.25(0.57,2.73)$ & 9.08 \\
\hline Solomon et $\mathrm{al}^{30}$ & $2.01(0.91,4.45)$ & 7.44 \\
\hline Sailasree et $\mathrm{al}^{29}$ & $1.37(0.08,22.17)$ & 0.68 \\
\hline Supic et $\mathrm{al}^{31}$ & $1.56(0.72,3.35)$ & 8.30 \\
\hline Tsai et $\mathrm{al}^{32}$ & $0.67(0.45,1.01)$ & 47.09 \\
\hline Vylliotis et $\mathrm{al}^{34}$ & $0.63(0.22,1.81)$ & 7.38 \\
\hline Bezerra et $\mathrm{al}^{27}$ & $2.87(0.17,47.30)$ & 0.41 \\
\hline Barbosa et al ${ }^{19}$ & $0.52(0.19,1.47)$ & 8.40 \\
\hline Miri-Moghaddam et al ${ }^{18}$ & $2.22(0.20,25.14)$ & 0.75 \\
\hline Overall $\left(I^{2}=21.3 \%, P=0.247\right)$ & $0.95(0.74,1.23)$ & 100 \\
\hline $\begin{array}{c} \\
0.0211\end{array}$ & & \\
\hline
\end{tabular}

Figure 2 Forest plot for the association between MTHFR C677T polymorphism and oral cancer risk under recessive model.

Notes: For each study, the estimated OR and its $95 \% \mathrm{Cl}$ are plotted with a box and a horizontal line. $\diamond$, pooled ORs and its $95 \% \mathrm{Cls}$.

Abbreviations: MTHFR, methylenetetrahydrofolate reductase; $\mathrm{OR}$, odds ratio; $\mathrm{Cl}$, confidence interval.

by Zhu et $\mathrm{al}^{23}$ in 2016, they suggested that MTHFR A1298C polymorphism was associated with decreased oral cancer risk. However, this association may not be convincible enough due to the relatively small sample; thus, it is necessary for us to update this research.
It should be noted that our stratified analyses revealed a borderline significant association of C677T polymorphism with a decreased oral cancer risk under the homozygous model in studies with hospital-based design. As the variant T alleles of MTHFR C677T polymorphism could reduce the

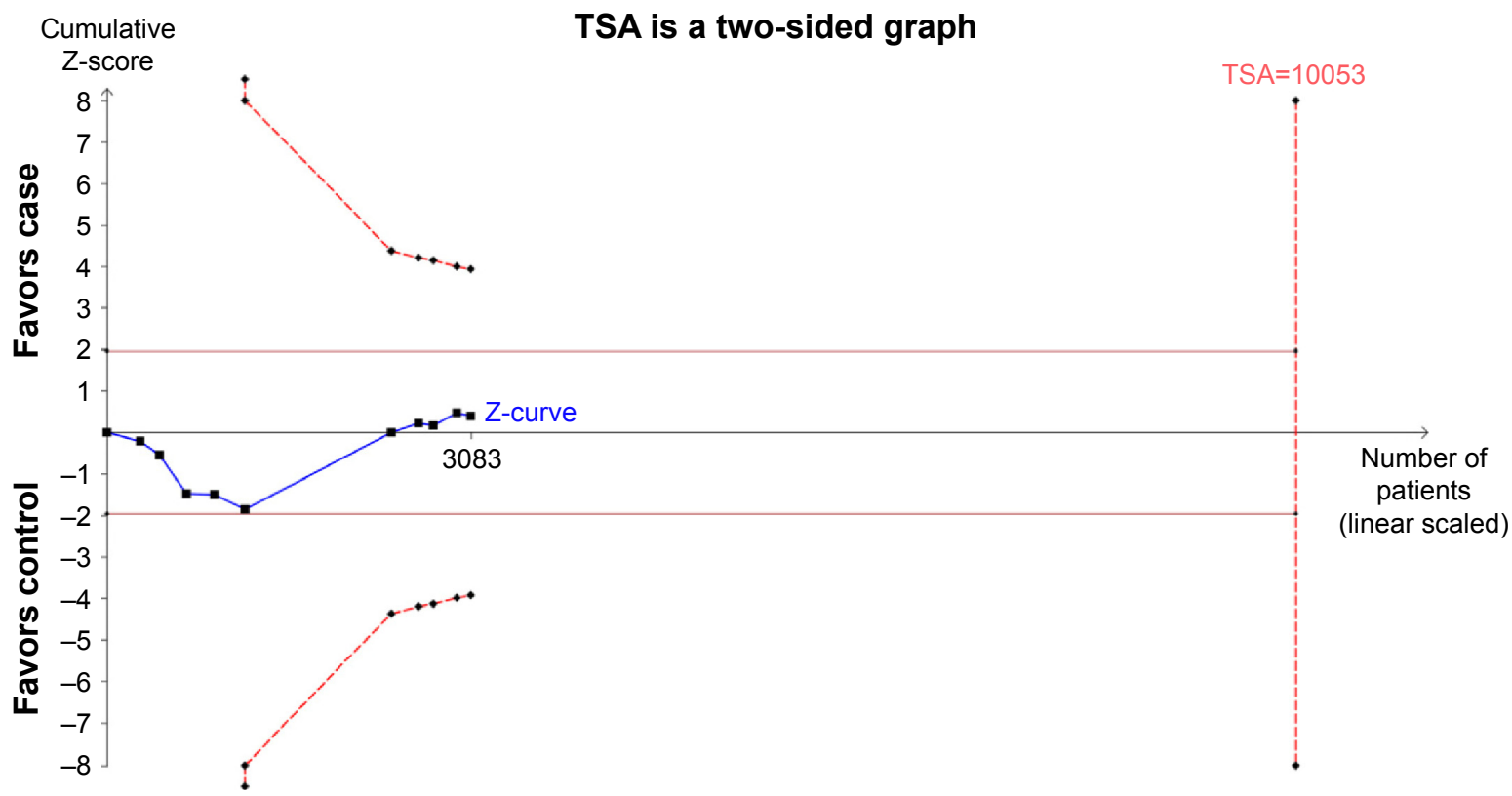

Figure 3 The required information size to demonstrate the association between MTHFR C677T polymorphism with oral cancer susceptibility.

Notes: The solid blue line is the cumulative Z-curve. The dashed inward-sloping line to the left represents the trial sequential monitoring boundaries.

Abbreviations: MTHFR, methylenetetrahydrofolate reductase; TSA, trial sequencing analysis. 
enzyme activity to nearly $50 \%$ compared to the wild-type $\mathrm{C}$ allele, we hypothesize that the reduced MTHFR activity will cause intracellular accumulation of 5,10-methylenetetrahydrofolate and result in a reduced incidence of DNA double-strand breakage. The reduced incidence of DNA double-strand breakage will maintain genetic stability and prevent oral cancer development. ${ }^{45}$ Besides, we also observed that there seem to be different results from Asian and Caucasian studies for the association between C677T polymorphism and oral cancer risk. The discrepant results may be explained by the fact that different races may modify the genetic effect on oral cancer risk.

Compared to the previous meta-analyses, we have undertaken some critical measurements to assure the credibility of our conclusion. The publication bias analysis and sensitivity analysis indicated a lack of publication bias and the stability of the results. We also employed TSA to test the conclusion reliability of this meta-analysis. However, the Z-curve failed to cross the trial monitoring boundary before reaching the required information size, which indicated that more samples were needed to clarify the correlation between MTHFR C677T polymorphism and oral cancer risk.

In spite of the merits, some weaknesses of the study should be pointed out. First, the statistical power of the meta-analysis was restricted because of relative small sample size. Second, the existence of between-study heterogeneity in some comparisons might compromise the reliability of conclusion. Third, only relevant studies in English and Chinese were included, some investigations in other languages might be omitted. Forth, due to the utilization of unadjusted ORs, we were only able to examine the association of interest by single-factor estimation from genetic perspective. The impacts of other risk factors for oral cancer, including lifestyles, environment exposures, and gene-environment interactions, are also needed to be considered. Finally, there were some inherent limitations due to the retrospective nature of meta-analysis, such as inconsistent qualities of the included studies, misclassified genotypes, incomplete histological details, and unwell-matched sources of controls.

\section{Conclusion}

Despite some limitations, this meta-analysis indicated that there was no association observed between the two MTHFR polymorphisms (C677T and A1298C) and oral cancer risk. Further in-depth and well-designed investigations with larger sample sizes are warranted to explore the correlation, since this null correlation estimated by conventional meta-analysis failed to get the conformation of TSA.

\section{Acknowledgment}

This study was supported by grants from Science and Technology Planning Project of Guangdong Province (2007B031514005).

\section{Disclosure}

The authors report no conflicts of interest in this work.

\section{References}

1. Torre LA, Bray F, Siegel RL, Ferlay J, Lortet-Tieulent J, Jemal A. Global cancer statistics, 2012. CA Cancer J Clin. 2015;65(2):87-108.

2. Blot WJ, McLaughlin JK, Winn DM, et al. Smoking and drinking in relation to oral and pharyngeal cancer. Cancer Res. 1988;48(11): 3282-3287.

3. Abbasi R, Ramroth H, Becher H, Dietz A, Schmezer P, Popanda O. Laryngeal cancer risk associated with smoking and alcohol consumption is modified by genetic polymorphisms in ERCC5, ERCC6 and RAD23B but not by polymorphisms in five other nucleotide excision repair genes. Int J Cancer. 2009;125(6):1431-1439.

4. Bravi F, Bosetti C, Filomeno M, et al. Foods, nutrients and the risk of oral and pharyngeal cancer. Br J Cancer. 2013;109(11):2904-2910.

5. Hou J, Gu Y, Hou W, et al. P53 codon 72 polymorphism, human papillomavirus infection, and their interaction to oral carcinoma susceptibility. BMC Genet. 2015;16:72.

6. Liao G, Wang Y, Zhou YQ, et al. Host genetic susceptibility to oral cancer: evidence from meta-analyses and pooled analyses. Oral Dis. 2014;20(7):644-649.

7. Giovannucci E, Rimm EB, Ascherio A, Stampfer MJ, Colditz GA, Willett WC. Alcohol, low-methionine - low-folate diets, and risk of colon cancer in men. J Natl Cancer Inst. 1995;87(4):265-273.

8. Pelucchi C, Talamini R, Negri E, et al. Folate intake and risk of oral and pharyngeal cancer. Ann Oncol. 2003;14(11):1677-1681.

9. Blount BC, Mack MM, Wehr CM, et al. Folate deficiency causes uracil misincorporation into human DNA and chromosome breakage: implications for cancer and neuronal damage. Proc Natl Acad Sci U S A. 1997;94(7):3290-3295.

10. Duthie SJ. Folic acid deficiency and cancer: mechanisms of DNA instability. Br Med Bull. 1999;55(3):578-592.

11. Niclot $\mathrm{S}$, Pruvot $\mathrm{Q}$, Besson $\mathrm{C}$, et al. Implication of the folate-methionine metabolism pathways in susceptibility to follicular lymphomas. Blood. 2006;108(1):278-285

12. Tang M, Wang SQ, Liu BJ, et al. The methylenetetrahydrofolate reductase (MTHFR) C677T polymorphism and tumor risk: evidence from 134 case-control studies. Mol Biol Rep. 2014;41(7):4659-4673.

13. Huang L, Deng D, Peng Z, et al. Polymorphisms in the methylenetetrahydrofolate reductase gene (MTHFR) are associated with susceptibility to adult acute myeloid leukemia in a Chinese population. Cancer Epidemiol. 2015;39(3):328-333.

14. Awwad N, Yousef AM, Abuhaliema A, Abdalla I, Yousef M. Relationship between genetic polymorphisms in MTHFR (C677T, A1298C and their haplotypes) and the incidence of breast cancer among Jordanian females - case-control study. Asian Pac J Cancer Prev. 2015; 16(12):5007-5011.

15. Yin G, Kono S, Toyomura K, et al. Methylenetetrahydrofolate reductase C677T and A1298C polymorphisms and colorectal cancer: the Fukuoka Colorectal Cancer Study. Cancer Sci. 2004;95(11):908-913.

16. He J, Liao XY, Zhu JH, et al. Association of MTHFR C677T and A1298C polymorphisms with non-Hodgkin lymphoma susceptibility: evidence from a meta-analysis. Sci Rep. 2014;4:6159.

17. Zhu J, Wu L, Kohlmeier M, Ye F, Cai W. Association between MTHFR C677T, MTHFR A1298C and MS A2756G polymorphisms and risk of cervical intraepithelial neoplasia II/III and cervical cancer: a metaanalysis. Mol Med Rep. 2013;8(3):919-927. 
18. Miri-Moghaddam E, Saravani S, Garme Y, Khosravi A, Bazi A, Motazedian J. Methylenetetrahydrofolate reductase C677T and A1298C gene polymorphisms in oral squamous cell carcinoma in south-east Iran. J Oral Pathol Med. 2016;45(2):96-100.

19. Barbosa A, Dos Santos M, de Podesta JR, et al. Polymorphisms in methylenetetrahydrofolate reductase and cystathionine beta-synthase in oral cancer - a case-control study in southeastern Brazilians. Braz J Otorhinolaryngol. 2016;82(5):558-566.

20. Zhuo X, Ling J, Zhou Y, Zhao H, Song Y, Tan Y. Polymorphisms of MTHFR C677T and A1298C association with oral carcinoma risk: a meta-analysis. Cancer Invest. 2012;30(6):447-452.

21. Jia J, Ma Z, Wu S. Positive association between MTHFR C677T polymorphism and oral cancer risk: a meta-analysis. Tumour Biol. 2014;35(5):4943-4948.

22. Xu Q, Niu Y, Hu X, Zhou J, Leng W. Association between MTHFR C677T polymorphism and oral cancer risk. J Clin Stomatol. 2013; 29(8):4.

23. Zhu F, Chen Y, Zou J, Cao Y, Gao Y, Qian H. MTHFR C677T and A1298C polymorphisms and oral cancer risk: an updated meta-analysis. Int J Clin Exp Med. 2016;9(9):18712-18720.

24. Xie S, Shan XF, Shang K, Xu H, He J, Cai ZG. Relevance of LIG4 gene polymorphisms with cancer susceptibility: evidence from a metaanalysis. Sci Rep. 2014;4:6630.

25. Vairaktaris E, Yapijakis C, Kessler P, et al. Methylenetetrahydrofolate reductase polymorphism and minor increase of risk for oral cancer. J Cancer Res Clin Oncol. 2006;132(4):219-222.

26. Galbiatti AL, Ruiz MT, Rodrigues JO, et al. Polymorphisms and haplotypes in methylenetetrahydrofolate reductase gene and head and neck squamous cell carcinoma risk. Mol Biol Rep. 2012;39(1):635-643.

27. Bezerra AM, Sant'Ana TA, Gomes AV, de Lacerda Vidal AK, Muniz MT. Tyms double (2R) and triple repeat (3R) confers risk for human oral squamous cell carcinoma. Mol Biol Rep. 2014;41(12): 7737-7742.

28. Capaccio P, Ottaviani F, Cuccarini V, Cenzuales S, Cesana BM, Pignataro L. Association between methylenetetrahydrofolate reductase polymorphisms, alcohol intake and oropharyngolaryngeal carcinoma in northern Italy. J Laryngol Otol. 2005;119(5):371-376.

29. Sailasree R, Nalinakumari KR, Sebastian P, Kannan S. Influence of methylenetetrahydrofolate reductase polymorphisms in oral cancer patients. J Oral Pathol Med. 2011;40(1):61-66.

30. Solomon PR, Selvam GS, Shanmugam G. Polymorphism in ADH and MTHFR genes in oral squamous cell carcinoma of Indians. Oral Dis. 2008;14(7):633-639.

31. Supic G, Jovic N, Kozomara R, Zeljic K, Magic Z. Interaction between the MTHFR C677T polymorphism and alcohol - impact on oral cancer risk and multiple DNA methylation of tumor-related genes. J Dent Res. 2011;90(1):65-70.

32. Tsai CW, Hsu CF, Tsai MH, et al. Methylenetetrahydrofolate reductase (MTHFR) genotype, smoking habit, metastasis and oral cancer in Taiwan. Anticancer Res. 2011;31(6):2395-2399.
33. Weinstein SJ, Gridley G, Harty LC, et al. Folate intake, serum homocysteine and methylenetetrahydrofolate reductase (MTHFR) C677T genotype are not associated with oral cancer risk in Puerto Rico. J Nutr. 2002;132(4):762-767.

34. Vylliotis A, Yapijakis C, Nkenke E, et al. Effect of thrombosis-related gene polymorphisms upon oral cancer: a regression analysis. Anticancer Res. 2013;33(9):4033-4039.

35. Friso S, Choi SW, Girelli D, et al. A common mutation in the 5,10methylenetetrahydrofolate reductase gene affects genomic DNA methylation through an interaction with folate status. Proc Natl Acad Sci US A. 2002;99(8):5606-5611.

36. Frosst $\mathrm{P}, \mathrm{Blom} \mathrm{HJ}$, Milos R, et al. A candidate genetic risk factor for vascular disease: a common mutation in methylenetetrahydrofolate reductase. Nat Genet. 1995;10(1):111-113.

37. Bai JL, Zheng MH, Xia X, Ter-Minassian M, Chen YP, Chen F. MTHFR C677T polymorphism contributes to prostate cancer risk among Caucasians: a meta-analysis of 3511 cases and 2762 controls. Eur J Cancer. 2009;45(8):1443-1449.

38. Taioli E, Garza MA, Ahn YO, et al. Meta- and pooled analyses of the methylenetetrahydrofolate reductase (MTHFR) C677T polymorphism and colorectal cancer: a HuGE-GSEC review. Am J Epidemiol. 2009;170(10):1207-1221

39. Wiemels JL, Smith RN, Taylor GM, et al; United Kingdom Childhood Cancer Study Investigators. Methylenetetrahydrofolate reductase (MTHFR) polymorphisms and risk of molecularly defined subtypes of childhood acute leukemia. Proc Natl Acad Sci U S A. 2001; 98(7):4004-4009.

40. Matsuo K, Suzuki R, Hamajima N, et al. Association between polymorphisms of folate- and methionine-metabolizing enzymes and susceptibility to malignant lymphoma. Blood. 2001;97(10):3205-3209.

41. Qi X, Ma X, Yang X, et al. Methylenetetrahydrofolate reductase polymorphisms and breast cancer risk: a meta-analysis from 41 studies with 16,480 cases and 22,388 controls. Breast Cancer Res Treat. 2010; 123(2):499-506.

42. Dong X, Wu J, Liang P, Li J, Yuan L, Liu X. Methylenetetrahydrofolate reductase C677T and A1298C polymorphisms and gastric cancer: a meta-analysis. Arch Med Res. 2010;41(2):125-133.

43. Jin F, Qu LS, Shen XZ. Association between the methylenetetrahydrofolate reductase C677T polymorphism and hepatocellular carcinoma risk: a meta-analysis. Diagn Pathol. 2009;4:39.

44. Goodman MT, McDuffie K, Hernandez B, et al. Association of methylenetetrahydrofolate reductase polymorphism C677T and dietary folate with the risk of cervical dysplasia. Cancer Epidemiol Biomarkers Prev. 2001;10(12):1275-1280.

45. Hagmar L, Brogger A, Hansteen IL, et al. Cancer risk in humans predicted by increased levels of chromosomal aberrations in lymphocytes: nordic study group on the health risk of chromosome damage. Cancer Res. 1994;54(11):2919-2922.
OncoTargets and Therapy

\section{Publish your work in this journal}

OncoTargets and Therapy is an international, peer-reviewed, open access journal focusing on the pathological basis of all cancers, potential targets for therapy and treatment protocols employed to improve the management of cancer patients. The journal also focuses on the impact of management programs and new therapeutic agents and protocols on
Dovepress

patient perspectives such as quality of life, adherence and satisfaction The manuscript management system is completely online and includes a very quick and fair peer-review system, which is all easy to use. Visit http://www.dovepress.com/testimonials.php to read real quotes from published authors. 